2. Let $\hat{H}^{n}(U, A) \cong \hat{H}^{n}(U, B)$ in two successive dimensions and for all subgroups but do not assume the isomorphisms induced by a module homomorphism. It would not be reasonable to expect isomorphisms for all $n$ and all subgroups. The following counterexample justifies our pessimism. Let $G=G_{p}\left(a, b: a^{3}=b^{7}=1, a b a^{-1}=b^{2}\right)$; let $A$ be $Z$ with trivial action and $B$ the result of dimension shifting down two steps. Then $\hat{H}^{q}(G, A ; 7)=\hat{H}^{q-2}(G, B ; 7)=0$ for $q=1,2,3,4,5$ and $\hat{H}^{6}(G, A ; 7)=\hat{H}^{4}(G, B ; 7) \neq 0$.

University of California, Berkeley

\title{
QUASI-INVERTIBLE PRIME IDEALS
}

\section{H. S. BUTTS}

In this note $R$ will denote a commutative ring with unit and a proper ideal of $R$ is an ideal of $R$ different from (0) and $R$. Nakano has shown that $R$ is a Dedekind domain, provided that every proper prime ideal of $R$ is invertible [1]. In [2], Krull defines a prime ideal $P$ to be quasi-invertible provided $P P^{-1}>P$, where $>$ denotes proper containment and $P^{-1}$ is the set of elements $x$ in the total quotient ring of $R$ such that $x P \subset R$. The purpose of this note is to prove that Nakano's result remains valid when invertible is replaced by quasiinvertible. Examples are known of rank-two valuation rings in which the maximal ideal is invertible-hence, in Nakano's result, prime cannot be replaced by maximal.

Lemma. If $P$ is an invertible prime ideal in $R$ then $\bigcap_{n} P^{n}$ is a prime ideal.

Proof. The proof is the same as that of the first part of Theorem 4 of $[1]$.

THEOREM. If every proper prime ideal of $R$ is quasi-invertible, then $R$ is a Dedekind domain.

Proof. If $R$ is a field there is nothing to prove. Let $M$ be an arbitrary proper maximal ideal of $R$ and denote by $R_{M}$ the quotient ring of $R$ with respect to $M$ (see [3, pp. 218-228]). Let $N$ denote the ideal consisting of the elements $x \in R$ such that there exists an element $m \notin M$ such that $m x=0$. Let $h$ be the natural homomorphism from

Received by the editors November 4, 1963. 
$R$ onto $\bar{R}=R / N$. If $T$ and $\bar{T}$ denote the total quotient rings of $R$ and $\bar{R}$, respectively, then $h$ may be continued to a homomorphism $f$ from $T$ into $\bar{T}$ (where $f(a / b)=h(a) / h(b)$ ). We may suppose that $\bar{R} \subset R_{M} \subset \bar{T}$ (see $[3])$.

Since $M$ is a quasi-invertible maximal ideal in $R$, then $M M^{-1}=R$. Therefore $\bar{R}=f\left(M M^{-1}\right)=f(M) f\left(M^{-1}\right)=h(M) f\left(M^{-1}\right)$, and, hence, $R_{M}=h(M) R_{M} f\left(M^{-1}\right) R_{M}$. It follows that the maximal ideal $h(M) R_{M}$ in $R_{M}$ is invertible. By the lemma, $\cap_{n}\left[h(M) R_{M}\right]^{n}$ is a prime ideal in $R_{M}$, and, therefore, there exists a prime ideal $P \subset M \subset R$ such that $h(P) R_{M}=\bigcap_{n} h\left(M^{n}\right) R_{M}$. Suppose $P \neq(0)$. If $P P^{-1} \nsubseteq M$ then $h\left(P P^{-1}\right) R_{M}$ $=R_{M}$ and it follows that the prime ideal $h(P) R_{M}$ is invertible. If $P P^{-1} \subset M$, then there exists a positive integer $n$ such that $P P^{-1} \subset M^{n}$ and $P P^{-1} \nsubseteq M^{n+1}$ since $P P^{-1}>P$. Since $M$ is invertible, there exists an ideal $Q$ in $R$ such that $Q M^{n}=P P^{-1}$ and $Q \nsubseteq M$. Hence $h\left(P P^{-1}\right) R_{M}$ $=h\left(Q M^{n}\right) R_{M}=h(Q) h\left(M^{n}\right) R_{M}=h\left(M^{n}\right) R_{M}$, and therefore, $h\left(P P^{-1}\right) R_{M}$ $=f\left(P P^{-1}\right) R_{M}=f(P) R_{M} f\left(P^{-1}\right) R_{M}=h(P) R_{M} f\left(P^{-1}\right) R_{M}=h\left(M^{n}\right) R_{M}$. This implies that the prime ideal $h(P) R_{M}$ is invertible. Thus, in either case, the prime ideal $h(P) R_{M}$ is invertible, which is a contradictionit is clear that an invertible proper prime cannot properly contain another invertible prime. It follows that $P=(0)$ and therefore $R_{M}$ is a domain in which proper prime ideals are maximal. Hence proper prime ideals are maximal in $R$ and therefore proper prime ideals are invertible in $R$. It follows that $R$ is a Dedekind domain (see [1]).

\section{REFERENCES}

1. Noboru Nakano, Über die Umkehrbarkeit der Ideale im Integritatsbereiche, Proc. Imp. Acad. Tokyo 19 (1943), 230-234.

2. W. Krull, Über den Aufbau des Nullideals in ganz abgeschlossenen Ringen mit Teilerkettensatz, Math. Ann. 102 (1929), 363-369.

3. O. Zariski and P. Samuel, Commutative algebra, Vol. 1, Van Nostrand, Princeton, N. J., 1958.

Louisiana State University 